\title{
The Econometrics and Some Properties of Separable Matching Models
}

\author{
Alfred Galichon* $\quad$ Bernard Salanié ${ }^{\dagger}$
}

December 28, 2016

\begin{abstract}
We present a class of one-to-one matching models with perfectly transferable utility. We discuss identification and inference in these separable models, and we show how their comparative statics are readily analyzed.
\end{abstract}

Keywords: sorting, matching, marriage market, gross substitutes.

JEL Classification: D3, J21, J23 and J31.

\section{Introduction}

Eugene Choo and Aloysius Siow's (2006) contribution has renewed interest in empirical applications of matching with perfectly transferable utility (TU). Unobserved heterogeneity in joint surplus is a paramount consideration in the specification of these models. Choo and Siow chose a separable multilogit model, which leads to highly tractable formulæ. But unobserved heterogeneity could originate from variation in tastes, from division of labor within the partners, and other sources. It is therefore important to allow for flexibility in

*Department of Economics, FAS, and Department of Mathematics, Courant Institute, New York University. Email: ag133@nyu.edu.

${ }^{\dagger}$ Department of Economics, Columbia University. Email: bs2237@columbia.edu. 
the stochastic specification of the joint surplus. Alfred Galichon and Bernard Salanié (2016) have explored a general class of models of bilateral matching which sets very few constraints on the distributions of unobserved heterogeneity beyond separability of the joint surplus. These separable models have a nicely convex and (usually) smooth structure that generates very useful econometric and analytic properties.

We start by summarizing our main results concerning identification and inference in separable models of one-to-one matching under $\mathrm{TU}^{1}$. We then show how in models with separable heterogeneity and full support, we can use the implicit function theorem and matrix algebra to get explict formulæ for any small change in the primitives of the model: arrival or departure of a mass of individuals of a given type, or changes in joint surplus. We illustrate the usefulness of our formulæ on a simple example.

\section{Separable models with full support}

In this paper we will call "men" and "women" the agents on both sides of the market, as is traditional; but our results apply more generally than in this implicit heterosexual marriage market à la Becker. We assume that agents on both sides of the market belong to continuous sets $\mathcal{I}$ and $\mathcal{J}$, which are partitioned into finite sets of types. A man $i \in \mathcal{I}$ has a type $x_{i} \in \mathcal{X}$ and a woman $j \in \mathcal{J}$ has a type $y_{j} \in \mathcal{Y}$, where $\mathcal{X}$ and $\mathcal{Y}$ are finite. The mass of men of type $x$ (resp. women of type $y$ ) is $n_{x}$ (resp. $m_{y}$ ). The distinction between types and identities is data-driven: while participants on the market are assumed to operate under perfect information, the analyst only observes the types $x$ and $y$. We also assume that joint surplus is separable:

$$
\tilde{\Phi}_{i j}=\Phi_{x_{i} y_{j}}+\varepsilon_{i y_{j}}+\eta_{j x_{i}}
$$

Separability excludes interactions between unobserved characteristics of $i$ and $j$ conditional on observed types $(x, y)$. As an example, let types describe education, as in Pierre-André

\footnotetext{
${ }^{1}$ Galichon-Salanié (2016) has detailed arguments, along with somehat weaker assumptions than we use here.
} 
Chiappori, Salanié, and Yoram Weiss (2016). Then separability does allow for unlimited unobserved heterogeneity in the way more-educated men value the education of their partners for instance; it rules out considerations like matching on physical characteristics, which certainly exists but may not be that relevant for the study of some economic questions at least.

We know from Chiappori-Salanié-Weiss (2016) and Galichon-Salanié (2016) that if $i$ and $j$ match then the man receives utility $U_{x_{i} y_{j}}+\varepsilon_{i y_{j}}$ and the woman receives utility $V_{x_{i} y_{j}}+\eta_{j x}$, where the terms $\boldsymbol{U}=\left(U_{x y}\right)$ and $\boldsymbol{V}=\left(V_{x y}\right)$ are endogenously determined at equilibrium so that $U_{x y}+V_{x y}=\Phi_{x y}$. A single man $i$ receives utility $\varepsilon_{i 0}$, while a single woman $j$ receives $\eta_{j 0}$. The interpretation of this result is simple: the $\varepsilon_{i y}$ of man $i$ has the same value for 11 women of type $y$, and since there is a continuum of them they will compete for it until the "price" of man $i$ fully incorporates it.

We now denote $\mathcal{X}_{0}=\mathcal{X} \cup\{0\}$ and $\mathcal{Y}_{0}=\mathcal{Y} \cup\{0\}$. We shall assume that the random vector $\varepsilon_{x}=\left(\varepsilon_{i y}\right) \in \mathbb{R}^{\mathcal{Y}_{0}}$ is distributed as $\mathbf{P}_{x}$ identically and independently across the population of men $i$ of type $x$; and we introduce $\mathbf{Q}_{y}$ in the same way for women. In this note we will also impose full support: for each $x \in \mathcal{X}, \mathbf{P}_{x}$ has a nonvanishing density on $\mathbb{R}^{\mathcal{Y}_{0}}$, and for each $y \in \mathcal{Y}, \mathbf{Q}_{y}$ has a nonvanishing density on $\mathbb{R}^{\mathcal{X}_{0}}$.

\section{Equilibrium and welfare}

When $\mathbf{P}_{x}$ and $\mathbf{Q}_{y}$ are Gumbel distributions for all $x$ and $y$, the model boils down to the model of Choo and Siow (2006). More generally, Galichon and Salanié (2016) introduce the convex functions

$$
G_{x}(\boldsymbol{U})=\mathbb{E}_{\mathbf{P}_{x}}\left[\max _{y \in \mathcal{Y}}\left\{U_{x y}+\varepsilon_{y}, \varepsilon_{0}\right\}\right] \text { and } H_{y}(\boldsymbol{V})=\mathbb{E}_{\mathbf{Q}_{y}}\left[\max _{x \in \mathcal{X}}\left\{V_{x y}+\eta_{x}, \eta_{0}\right\}\right],
$$

and

$$
G(\boldsymbol{U})=\sum_{x \in \mathcal{X}} n_{x} G_{x}(\boldsymbol{U}) \text { and } H(\boldsymbol{V})=\sum_{y \in \mathcal{Y}} m_{y} H_{y}(\boldsymbol{V})
$$


Galichon and Salanié (2016, Theorem 2) show that $\boldsymbol{U}$ minimizes the expression $G(\boldsymbol{U})+$ $H(\boldsymbol{\Phi}-\boldsymbol{U})$. Under separability and full support, the functions $G$ and $H$ are strictly convex and twice differentiable, and the first-order conditions characterize the unique equilibrium:

$$
\nabla G(\boldsymbol{U})=\nabla H(\boldsymbol{\Phi}-\boldsymbol{U})
$$

These conditions are easily interpreted. By the Daly-Zachary-Williams theorem, the mass of men of type $x$ wishing to match with women of type $y \in \mathcal{Y}$ given a vector $\boldsymbol{U}$ is $\boldsymbol{U}$ is $\mu_{x y}=\partial G(\boldsymbol{U}) / \partial U_{x y}$. Similarly, the number of women of type $y$ wishing to match with men of type $x \in \mathcal{X}$ is $\mu_{x y}=\partial H(\boldsymbol{V}) / \partial V_{x y}$. In equilibrium, the two quantities $\nabla G(\boldsymbol{U})$ and $\nabla H(\boldsymbol{V})$ must coincide; and since $\boldsymbol{U}+\boldsymbol{V}=\mathbf{\Phi}, \boldsymbol{U}$ is determined in equilibrium by (3.1). Also note that the expected utility of the average man of type $x$ is $u_{x}=G_{x}(\boldsymbol{U})$ in equilibrium. Galichon-Salanié (2016, section 5) details several approaches to computing the equilibrium efficiently. The convexity and smoothness of the problem make it very tractable numerically.

\section{Identification and Inference}

Convex duality is the key to the approach in Galichon and Salanié (2016). Remember that given any function $f(a)$, its Legendre-Fenchel transform is the function $f^{*}$ such that

$$
f^{*}(b)=\sup _{a}(a \cdot b-f(b)) .
$$

The function $f^{*}$ may be badly-behaved: it may take infinite values, for instance. But since $f^{*}$ is the supremum of linear functions of $b$, it is convex. And if $f$ is convex, it is the Legendre-Fenchel transform of $f^{*}$; and if $f$ and $f^{*}$ are strictly convex, then

$$
b=\nabla f(a) \text { iff } a=\nabla f^{*}(b) .
$$

Let us first apply this "convex inversion formula" to the strictly convex function $f=G$ :

$$
\boldsymbol{\mu}=\nabla G(\boldsymbol{U}) \text { iff } \boldsymbol{U}=\nabla G^{*}(\boldsymbol{\mu})
$$

Given a full specification for the distributions $\mathbf{P}_{x}$, the function $G$ can be computed, and its Legendre-Fenchel transform too. Feeding the observed matching patterns into $\boldsymbol{U}=\nabla G^{*}(\boldsymbol{\mu})$ 
directly identifies $\boldsymbol{U}$. Proceeding in the same way with $f(\boldsymbol{U})=H(\boldsymbol{\Phi}-\boldsymbol{U})$ identifies $\boldsymbol{\Phi}-\boldsymbol{U}=\nabla H^{*}(\boldsymbol{\mu}) ;$ and adding up,

$$
\boldsymbol{\Phi}=\nabla G^{*}(\boldsymbol{\mu})+\nabla H^{*}(\boldsymbol{\mu})
$$

which identifies the joint surplus $\mathbf{\Phi}$ from the (assumed) knowledge of the distributions $\mathbf{P}_{x}$ and $\mathbf{Q}_{y}$.

This is "conditional unrestricted identification": the joint surplus is identified without any prior restriction if the analyst somehow knows the distribution of unobserved heterogeneity. If for instance these distributions are only assumed to be known up to scale, then in order to achieve point identification of the joint surplus the analyst will need to impose restrictions on it. There is an unavoidable trade off here, which can be alleviated by pooling data from several markets and assuming some common features across markets ${ }^{2}$.

Once identification is achieved, inference is straightforward. It can be based directly on the equations above, or proceed via maximum likelihood, or by matching moments of some basis functions. The latter method is based on a linear expansion

$$
\Phi_{x y}(\boldsymbol{\lambda})=\lambda \cdot \phi_{x y}
$$

where $\phi$ is a vector of basis functions ${ }^{3}$. Galichon and Salanié (2016) show that finding the parameter vector $\boldsymbol{\lambda}$ that matches the observed comoments $\hat{\boldsymbol{C}}=\hat{E} \boldsymbol{\phi}$ gives a consistent estimator.

\section{Comparative Statics}

The separable structure of the problem naturally generates a number of comparative statics results that extend those obtained by Colin Decker et al. (2012) and Bryan Graham (2013) for the Choo and Siow model. Our assumptions on the unobserved heterogeneity yield enough smoothness and convexity that simple formulæ can be obtained.

\footnotetext{
${ }^{2}$ Chiappori, Salanié and Weiss (2016) gives an example, with an heteroskedastic version of the Choo and Siow model.

${ }^{3}$ For instance, a simple "assortative matching basis function" would be $\mathbb{1}(x=y)$.
} 
Take a well-known result: in two-sided matching models, the arrival of newcomers on one side of the market hurts all participants on the same side of the market, and benefits all participants on the opposite side of the market. This was proved by Alexander Kelso and Vincent Crawford (1982, Theorem 5) for a many-to-one matching model under a gross substitutes assumption; by David Gale and Marilda Sotomayor (1985, Theorem 2) for the NTU marriage model; and by Gabrielle Demange and Gale (1985, Corollary 3) for a general class of one-to-one models with transfers. But all of these proofs are purely qualitative. With separable models, it is easy to make these results quantitative, and more generally to analyze the effects of small changes in the primitives.

The functions $G$ and $H$ are not only twice differentiable and strictly convex: they are also submodular. The economic interpretation is straightforward. Given differentiability, the submodularity of $G$ requires that $\partial G^{2} / \partial U_{x y} \partial U_{x^{\prime} y^{\prime}} \leq 0$ for all $\left(x^{\prime}, y^{\prime}\right) \neq(x, y)$. But since $\mu_{x y}=\partial G_{x}(\boldsymbol{U}) / \partial U_{x y}$, this simply says that $\partial \mu_{x y} / \partial U_{x y^{\prime}} \leq 0$ : if alternative $y^{\prime}$ becomes more attractive, alternative $y$ will be less demanded at equilibrium. This is, of course, a gross substitutes property.

To state our results, we need some more notation:

- we define matching ratios by $\mu_{x y}=\mu_{y \mid x}^{M} n_{x}=\mu_{x \mid y}^{W} m_{y}$; note that $\sum_{y} \mu_{y \mid x}^{M}=1-\mu_{0 \mid x}^{M}$ and $\sum_{x} \mu_{x \mid y}^{W}=1-\mu_{0 \mid y}^{W}$.

- we denote $\boldsymbol{T}=\left(D^{2} G(\boldsymbol{U})+D^{2} H(\boldsymbol{\Phi}-\boldsymbol{U})\right)^{-1}$ the inverse of the sum of the Hessians of $G$ and $H$ at the equilibrium $\boldsymbol{U}$ (the sum is invertible since $G$ and $H$ are strictly convex.)

- We use specific notation for some of its blocks; for instance, we denote $\boldsymbol{T}_{x, \cdot y}$ the matrix $\boldsymbol{A}$ with elements $A_{t z}=T_{x t, z y}$. 


\subsection{General results for separable models}

The primitives of the model are $\boldsymbol{\theta}=(\boldsymbol{n}, \boldsymbol{m}, \boldsymbol{\Phi})$. The equilibrium $\boldsymbol{U}$ is determined by $\nabla G(\boldsymbol{U})=\nabla H(\mathbf{\Phi}-\boldsymbol{U})$. Taking differentials, for all $x$ and $y$ we have

$$
\left\{D^{2} G(\boldsymbol{U})+D^{2} H(\boldsymbol{\Phi}-\boldsymbol{U})\right\} d \boldsymbol{U}=\frac{\partial^{2} H(\boldsymbol{\Phi}-\boldsymbol{U})}{\partial \boldsymbol{V} \partial \boldsymbol{m}} d \boldsymbol{m}-\frac{\partial^{2} G(\boldsymbol{U})}{\partial \boldsymbol{U} \partial \boldsymbol{n}} d \boldsymbol{n}+D^{2} H(\boldsymbol{\Phi}-\boldsymbol{U}) d \boldsymbol{\Phi} .
$$

Given strict convexity, the Hessians are negative definite, and the matrix $D^{2} G(\boldsymbol{U})+$ $D^{2} H(\boldsymbol{\Phi}-\boldsymbol{U})$ is invertible. Therefore we can write

$$
d \boldsymbol{U}=\boldsymbol{T} \boldsymbol{R} d \boldsymbol{\theta}
$$

where $\boldsymbol{R} d \boldsymbol{\theta}$ denotes the right-hand side of (5.1). Now since both $G$ and $H$ are submodular and strictly convex, $D^{2} G$ and $D^{2} H$ are Stieltjes matrices ${ }^{4}$, and so is their sum. By a classical result on Stieltjes matrices (see e.g. Golub and Van Loan 2013, lemma 11.5.1), all entries of $\boldsymbol{T}$ are nonnegative; and any change in $\boldsymbol{\theta}$ such that $\boldsymbol{R} d \boldsymbol{\theta}$ is a non-negative vector can only increase the equilibrium $U_{x y}$. Moreover, the average welfare of men of type $x \in \mathcal{X}$ is given by $u_{x}=G_{x}(\boldsymbol{U})$, and

$$
d u_{x}=\sum_{y \in \mathcal{Y}} \frac{\partial G_{x}}{\partial U_{x y}} d U_{x y}=\sum_{y \in \mathcal{Y}} \mu_{y \mid x}^{M} d U_{x y}
$$

so that any such change $\boldsymbol{R} d \boldsymbol{\theta} \geq 0$ can only increase the average expected utilities of men of any type.

Applying this to small changes in population sizes $\boldsymbol{n}$ and $\boldsymbol{m}$ yields very simple formul $\mathfrak{x}^{5}$ :

$$
\begin{aligned}
\frac{\partial u_{x}}{\partial n_{x^{\prime}}} & =\boldsymbol{\mu}_{\cdot \mid x^{\prime}}^{M} \boldsymbol{T}_{x \cdot, x^{\prime} \cdot \boldsymbol{\mu}_{\cdot \mid x^{\prime}}^{M} \leq 0}^{M} \leq 0 \\
\frac{\partial u_{x}}{\partial m_{y^{\prime}}} & =\boldsymbol{\mu}_{\cdot \mid x^{\prime}}^{M} \boldsymbol{T}_{x \cdot, \cdot y^{\prime}} \boldsymbol{\mu}_{\cdot \mid y^{\prime}}^{W} \geq 0 .
\end{aligned}
$$

The signs of the entries is a direct consequence of the non-negativity of all elements of $\boldsymbol{T}$; it was already known, but now we can easily compute the value of these local effects. In

\footnotetext{
${ }^{4}$ That is, they are positive definite with non-positive off-diagonal terms.

${ }^{5}$ The online appendix has the detail of these calculations.
} 
addition, it is easy to prove that

$$
\frac{\partial u_{x}}{\partial \Phi_{x^{\prime} y^{\prime}}}=\mu_{x y^{\prime}} \boldsymbol{T}_{x y^{\prime}, y^{\prime}} \frac{\partial^{2} H_{y^{\prime}}}{\partial V_{x^{\prime} y^{\prime}} \partial V_{\cdot y^{\prime}}} .
$$

Since $H_{y^{\prime}}$ is strictly convex and is submodular, the vector of second derivatives in this expression has one positive term, while all others are non-positive. Given the non-negativity of all elements of $\boldsymbol{T}$, an increase in any element $\Phi_{x^{\prime} y^{\prime}}$ of the joint surplus should reduce (resp. increase) the expected utility of men whom women of type $y^{\prime}$ see as good (resp. bad) substitutes of type $x^{\prime}$. These effects are larger for the men who are more likely to marry women of type $y^{\prime}$.

More generally, for any small change in the primitives of the model, we recover $d u_{x}=$ $\sum_{y} \mu_{y \mid x}^{M} d U_{x y}$ from the solution of the system

$$
\begin{gathered}
n_{x} \sum_{t} \frac{\partial^{2} G_{x}}{\partial U_{x y} \partial U_{x t}} d U_{x t}+m_{y} \sum_{z} \frac{\partial^{2} H_{y}}{\partial V_{x y} \partial V_{z y}} d U_{z y} \\
=\mu_{x y} d \log \frac{m_{y}}{n_{x}}+m_{y} \sum_{z} \frac{\partial^{2} H_{y}}{\partial V_{x y} \partial V_{z y}} d \Phi_{z y} .
\end{gathered}
$$

While $G_{x}$ and $H_{y}$ are functions of $\boldsymbol{U}$, using the Legendre-Fenchel transform we have $\boldsymbol{\mu}=$ $\nabla G^{*}(\boldsymbol{\mu})+H^{*}(\boldsymbol{\mu})$. Hence all of the elements of (5.4) can be computed from the observed data, given a structure $(\boldsymbol{\Phi}, \boldsymbol{n}, \boldsymbol{m})$.

\subsection{A one-type model}

For a drastically simple illustration, suppose that there is only one type of men and one type of women: $|X|=|Y|=1$. We simplify the notation by dropping the "1" subscripts, so that

$\Phi$ denotes $\Phi_{11}$ for instance. Equilibrium in this model consists in a number of marriages $\mu$, and associated expected utilities $u$ and $v$.

Now $G(U)=n E_{\mathbf{P}} \max \left(U+\varepsilon, \varepsilon_{0}\right)$. Let us denote $\left(F_{P}, f_{P}\right)$ the cdf and pdf of $\left(\varepsilon_{0}-\varepsilon\right)$ under $\mathbf{P}$; and define $k_{P}(t)=f_{P}\left(F_{P}^{-1}(t)\right)$. Then $G^{\prime}(U)=n F_{P}(U)$ and $G^{\prime \prime}(U)=n f_{P}(U)$. Using similar notation for $\mathbf{Q}$, the equilibrium $U$ and the number of marriages $\mu$ are given by $\mu=n F_{P}(U)=m F_{Q}(\Phi-U)$. Identification is straightforward: given $\mathbf{P}$ and $\mathbf{Q}$, solving 
these equations for $\Phi$ gives

$$
\Phi=F_{P}^{-1}\left(\frac{\mu}{n}\right)+F_{Q}^{-1}\left(\frac{\mu}{m}\right) .
$$

Moving to comparative statics, (5.4) becomes

$$
d U=T\left(\mu d \log \frac{m}{n}+m k_{Q}\left(\frac{\mu}{m}\right) d \Phi\right)
$$

with $T=1 / S$ and $S=n k_{P}(\mu / n)+m k_{Q}(\mu / m)$. Since $d u=(\mu / n) d U$, the change in the expected utilities of the average man follows directly, and so does the change in the number of marriages since $d \mu=F_{P}(U) d n+n f_{P}(U) d U$ :

$$
\begin{aligned}
& d u=T \frac{\mu}{n}\left(\mu d \log \frac{m}{n}+m k_{Q} d \Phi\right) \\
& d \mu=T\left(\mu\left(m k_{Q} d \log n+n k_{P} d \log m\right)+n m k_{P} k_{Q} d \Phi\right) .
\end{aligned}
$$

Take a small change $(d n, d m)$ in the sizes of the populations of men and of women. The resulting log-change $d \log \mu$ in the number of marriages will be a weighted average of the log-changes in $n$ and in $m$. More interestingly,

the changes in expected utilities of men and women directly reflect the change in the sex ratio $n / m$; and so do the changes in the percentage of singles in each gender.

If only the joint surplus of each marriage changes, by $d \Phi$, then

the number of marriages $\mu$ changes by a fraction $0<s<1$ of $d \Phi / 2$.

Assume moreover $\left(\varepsilon_{0}-\varepsilon\right)$ and $\left(\eta_{0}-\eta\right)$ have the same distribution, with cdf $F$ and pdf $f$; and let it be symmetric around 0 and log-concave. Then $0<U<\Phi / 2$ and $v>u>0$ if the sex ratio is unfavorable to men, $n<m$. Log-convavity gives us $k_{P}(\mu / n)<k_{Q}(\mu / m)$, so that $n k_{P}<m k_{Q}$ and $T n k_{P}<1 / 2<T m k_{Q}$. Therefore

the number of marriages is more elastic to the size of the smaller population. 


\section{References}

Chiappori, P.-A., Salanié, B. and Y. Weiss (2016): "Partner Choice, Investment in Children, and the Marital College Premium", mimeo.

Choo, E., and A. Siow (2006): "Who Marries Whom and Why," Journal of Political Economy, 114, 175-201.

Decker, C., E. Lieb, R. McCann, and B. Stephens (2012): "Unique Equilibria and Substitution Effects in a Stochastic Model of the Marriage Market," Journal of Economic Theory, 148, 778-792.

Demange, G. and D. Gale (1985): "The Strategy Structure of Two-Sided Matching Markets ", Econometrica, 53, 873-888.

Gale, D. and M. Sotomayor (1985): "Some Remarks on the Two-sided Matching Model", Discrete Applied Mathematics, 11, 223-232.

Galichon, A., and B. Salanié (2016): "Cupid's Invisible Hand: Social Surplus and Identification in Matching Models," working paper.

Golub, G. and Van Loan, C. (2013). Matrix computations. 4th edition. Johns Hopkins.

Graham, B. (2013): "Comparative static and computational methods for an empirical one-to-one transferable utility matching model". Structural Econometric Models 31, 153181.

Kelso, A. and V. Crawford (1982): "Job Matching, Coalition Formation, and Gross Substitutes", Econometrica, 50, 1483-1504. 